This is an Accepted Manuscript of an article published by Taylor \& Francis in Materials and Manufacturing Processes on 20/10/16, available online: http://www.tandfonline.com/doi/ full/10.1080/10426914.2016.1221092 


\title{
Production of high precision micro metallic components by electroforming process
}

\author{
Jian Liu ${ }^{1}$ *, Hany Hassanin ${ }^{2}$, Zhenyu Ni ${ }^{1}$, Yi Yang ${ }^{1}$, Gang Yang ${ }^{1}$, Kyle Jiang ${ }^{3}$ * \\ ${ }^{1}$ School of Manufacturing Science and Engineering, Sichuan University, Chengdu, \\ 610065, China. \\ ${ }^{2}$ School of Mechanical and Automotive Engineering, Kingston University, London, \\ SW153DW, UK. \\ ${ }^{3}$ School of Mechanical Engineering, University of Birmingham, Birmingham, B15 \\ 2TT, $U K$
}

\begin{abstract}
In this study nickel (Ni) micro components with high precision were produced by electroforming using a soft mould produced by softlithgrahpy and photolithgraphy techniques. The effects of current densities on the microstructures and mechanical properties of the fabricated micro components were studied. It is found that a high precision soft mould was sucessfully fabricated and micro Ni gears with sharp walls and fine features were produced. A feature size as small as approximately $70 \mu \mathrm{m}$, a height of $380 \mu \mathrm{m}$ and a pitch diameter of $3 \mathrm{~mm}$ were achieved for the gear. The densities of the gears electroformed are found to be within the range between 5.24 and $2.95 \mathrm{~g} / \mathrm{cm}^{3}$ when current densities utilized vary between 50 and $10 \mathrm{~mA} / \mathrm{cm}^{2}$. A lower current density leads to a finer microstructure and a larger value of microhardness. Meanwhile, by changing the current density from 50 to 10 $\mathrm{mA} / \mathrm{cm}^{2}$, the grain size decreases from around 50 to $30 \mathrm{~nm}$ while the microhardness increases from 220 to 410. In addition, the preferred orientation of the Ni matrix changes from (111) to (200) or (220) with the decreasing current density.
\end{abstract}

Introduction: The last decade has witnessed a tremendous development of microelectronic technologies and the fabrication of micro components with specific

\footnotetext{
* Corresponding author: E-mail address: liujian@ scu.edu.cn; * * Corresponding author: E-mail address:

K.Jiang@bham.ac.uk. Hany Hasssinin contributes to the work equally as the first author.
} 
mechanical or functional properties offers possibilities for a diverse range of applications [1-7]. In particular, metallic miniature parts already find applications in high sensitive temperature transducers and pressure sensors, high precision resistors and capacitors, wearable biomedical devices and ultra high speed inkjet printers. In order to produce high precision metallic or ceramic micro components, various types of fabrication techniques have been developed and optimized in recent years. Micro system technology invented decades ago can be able to produce micro components with feature size from nanometer to millimeter scale and is widely used in many fields [8]. X-ray lithography, as a well established micro fabrication technique can introduce high energy density to produce high precision microstructures on polymeric materials [9]. However, the use of the X-ray source usually incurs high cost and prevents it from further applications. Based on X-ray lithography, LIGA process was developed by combining the electroforming technique and already utilized to successfully create structures with high aspect ratio[10]. Nonetheless, the process often involves the sophisticated facilities and suffers from the low productivity. Micro electrodischarge machining $(\mu \mathrm{EDM})$ have been developed for years and can efficiently fabricate a variety of miniature products from an enormous range of metallic materials or composites [11-13]. However, $\mu \mathrm{EDM}$ is only effective in processing materials with adequate conductivity. To realize the removal of the nonconductive materials, laser micro machining was proposed and used to fabricate microstructures and micro components in an effective way. Despite its wide application there is always a need to improve the technique for producing smooth and fine surfaces. Micro metal injection moulding ( $\mu \mathrm{MIM})$ developed from MIM is considered a promising and economical way to mass produce micro components [14-17]. Nevertheless, the process chain of the MIM which involves powder mixing, injection moulding, debinding and sintering, is time consuming and environmentally unfriendly.

Electroforming is widely employed to successfully produce micro components of metallic materials [18] and careful control of the deposition rate of the metallic ions during the electroforming process can allow the formation of micro components with extraordinarily high precision[19]. In particular, the precision of the micro components is closely associated with that of the mould. So far, the mould for the 
electroforming process has been fabricated by LIGA or laser micro machining process, however, these methods are either too costly or can not satisfactorily achieve the desired precision. In order to improve the precision, combined techniques involving the photolithography and soft lithography offers a feasible alternative. The main advantage of the photolithography over other techniques is that much higher precision can be achieved and high quality microstructures can be fabricated during the process [2]. Meanwhile, the soft lithography allows the production of the replicas of the mould with fine features.[8, 20-21].

In the paper, high precision nickel $(\mathrm{Ni})$ micro components were produced by electroforming using an elastomeric soft mould prepared by soft lithography and photolithography techniques. The effects of current densities on the microstructures and mechanical properties of the prepared micro components were studied. The shape and the surface morphologies of the micro components were examined by the scanning electron microscope (SEM) and atomic force microscopy (AFM). Phase composition and grain size were determined by the x-ray diffraction (XRD). The mechanical properties were evaluated by measuring the relative densities and hardness of the samples.

\section{Materials and methods}

A master mould with shape of a high precision micro gear was produced on SU-8 photoresist using photolithography. The fabrication for the master mould is as follows: (i) directly cast appropriate amount of the photoresist on a silicon substrate and bake it for 3 hours at $65{ }^{\circ} \mathrm{C}$ and 34 hours at $95^{\circ} \mathrm{C}$ for $34 \mathrm{~h}$; (ii) have the SU-8 photoresist exposed to ultraviolet with a high exposure energy of $9000 \mathrm{~mJ} / \mathrm{cm}^{2}$ and the aid of filters (iii) post-bake for $5 \mathrm{~min}$ at $65{ }^{\circ} \mathrm{C}$ and for $15 \mathrm{~min}$ at $95{ }^{\circ} \mathrm{C}$; (iv) develop the baked resist in EC solvent [22-23]. The master mould fabricated was then used for the fabrication of soft moulds. The soft mould was prepared by mixing an elastomer and a curing agent (Dow Corning, Midland, MI) with a volume ratio of 10 to 1 and pouring the mixture on the master mould. After the mixture was cured, the polydimethylsiloxane (PDMS) soft mould was manually separated from the master mould.

The PDMS soft mould was coated with a gold layer using a gold sputtering machine and used as the cathode while a Ni plate was utilized as the anode. The Ni electrolyte used is made of boric acid (25-35 g/L), Ni chloride (8-12 g/L) and Ni (85-95 g/L) (PMD Chemicals Ltd, UK). Electroforming was performed to produce the Ni micro 
gears at a current density of 50,25 and $10 \mathrm{~mA} / \mathrm{cm}^{2}$ respectively and the corresponding deposited samples are denoted as $\mathrm{Ni}-1, \mathrm{Ni}-2$ and $\mathrm{Ni}-3$. After the electroforming, the micro gears were manually separated from the PDMS mould. Surfaces of the PDMS mould and micro Ni gears were examined using AFM and SEM. The crystal orientation and grain size were determined by XRD. The densities of the fabricated gears were measured by Archimedes' method. Microhardness was obtained by introducing $100 \mathrm{~g}$ load on the surface of the components for 15 seconds.

\section{Results and discussion}

The prepared master mould is shown in Figure 1. It can be seen that a mould of a micro gear with a height of $700 \mu \mathrm{m}$ and straight walls was successfully produced. The achievement of such a height of the micro gear is closely associated with the processing parameters. It is suggested that the exposure of the master mould to the ultraviolet is the critical step to fabricate vertical walls and usually a higher exposure energy can produce a sharper side walls [8]. In particular, SU-8 photoresist is rather sensitive to the wave length of the UV light. The increase of the wave length can considerably decrease the chance of the photoresist to absorb the energy of the light. In particular, when a wave length higher than $420 \mathrm{~nm}$ is selected, no absorbance of the light would occur while within $320 \mathrm{~nm}$ UV light would be completely absorbed [24]. In this work, a high energy density of $9000 \mathrm{~mJ} / \mathrm{cm}^{2}$ along with a filter was selected to ensure the production of a microcomponent with a large thickness and high precision. The high energy density selected can favor the crosslink reaction within the SU-8 photoresist and the use of the filter can allow only the wave length of near $320 \mathrm{~nm}$ to pass through and prevent the occurrence of T-topping effect, leading to the formation of straight and sharp walls.

A PDMS soft mould replica is shown in Figure 2 after the softlithography process and it can be seen that the small features of the master mould are well retained in the soft mould. PDMS is a particularly well-known for its usual rheological properties. It can flow freely in a liquid state to precisely replicate the details of the master mould and when it is cured, it can be imparted with a very high strength and can be utilized frequently for the subsequent electroforming process. AFM images of the master mould, the soft mould as prepared and coated with a layer of gold particles are given in Figure 3. As is shown, surface roughness of the soft mould is similar to that of the master mould, implying a high precision soft mould is successfully produced while 
the surface roughness of the soft mould coated with gold particles is almost ten times more than that of the master mould, indicating formation of a gold coating with thickness larger than $100 \mathrm{~nm}$.

Figure 4 shows SEM images of a typical electroformed Ni micro gear. It can be seen that micro gears display good shape profiles retaining the fine features and the sharp edges. The minimum feature size of the micro gear produced is approximately $70 \mu \mathrm{m}$. To further analyze the microstructures of the micro gears, the surfaces of micro gears electroformed at the varied current densities were then examined by XRD and high resolution SEM. The corresponding results are given in Figure 5 and it is noted that a lower current density leads to formation of a finer microstructure of the $\mathrm{Ni}$. In particular, a lower current density can help prevent the agglomeration of grains and formation of large particle sizes, resulting in a smoother surface morphology and better surface quality of the electroformed samples. It should be noted that edge effect is usually present in the electroplating process and current is prone to concentrating on the edges or corners of cathodes, leading to the formation of samples with non-uniform thickness and surface morphologies. An auxiliary electrode or adjustment of current densities was often employed to achieve a more uniform current distribution around cathodes [25]. In this study, varied current densities have been attempted and a relatively uniform thickness and a smooth surface are achieved using a low current density in a continuously agitated electroplating bath.

The XRD pattern of the Ni gear prepared at a higher current density exhibits sharper peaks as compared to that at a lower current density and the peak width of the $\mathrm{Ni}$ prepared at a lower current density is broader than that at a higher current density. It is reported that the grain sizes determined by XRD method are very consistent with those observed by transmission electron microscopy [26]. Grain sizes of the deposited $\mathrm{Ni}$ are therefore derived from the XRD results using the following equation [27]:

$$
Q=K \lambda / \beta \cos \theta
$$

Where $\theta$ is the diffraction angle, $\beta$ is the full-width at half-maximum, $\lambda$ is the wave length of the incident x-ray, $K$ is the Scherrer constant, $Q$ is the average grain size. 
Grain sizes of the Ni gears electrodeposied at varied current densities are shown in Figure 6a. It is evident a lower current density can result in the formation of $\mathrm{Ni}$ with smaller grain size and when the current density is decreased from 50 to $10 \mathrm{~mA} / \mathrm{cm}^{2}$, the grain sizes of the $\mathrm{Ni}$ are found to decrease from around 50 to $30 \mathrm{~nm}$. Hardness measured shows the inverse trend with the current density (Fig 6a). A high hardness of 410 is achieved by using a lower current density of $10 \mathrm{~mA} / \mathrm{cm}^{2}$. On the other hand, the density of the electrformed gear exhibits a moderate decrease initially from 5.24 to $2.95 \mathrm{~g} / \mathrm{cm}^{3}$ when the current density decreases from 50 to $25 \mathrm{~mA} / \mathrm{cm}^{2}$ and a significant increase to $4.05 \mathrm{~g} / \mathrm{cm}^{3}$ when the current density as low as $10 \mathrm{~mA} / \mathrm{cm}^{2}$ is used. The variation in density of the electroformed sample is attributed to both the deposition rate of $\mathrm{Ni}$ ions and the bubbles generated in electroplating practices. It is expected there is an optimum current density where a relatively high deposition rate can be achieved and bubbles formed would be readily released, leaving no pores in the electroformed samples and leading to a high density. Note that both a small grain size and a high density associated with the current density can contribute to a high hardness of the electrodeposited Ni.

To further analyse the microstructure, the XRD results were used to determine the preferred orientations of the matirx grain and the corresponding texture coefficients of varied crystal planes were derived based upon the equation below [28-29]:

$$
T c_{(h k l)}=\sum I_{0(h k l)} / I_{0(h k l)} \times I_{(h k l)} / \sum I_{(h k l)}
$$

Where $(h k l)$ and $I$ represent the crystal planes and the intensity of the reflection respectively. $O$ indicates the intensity of the reflection for the standard sample. Texture coefficients of the matrix microstructures are compared and given in Figure $6 \mathrm{c}$. It shows that $\mathrm{Ni}$ electrodeposited at $50 \mathrm{~mA} / \mathrm{cm}^{2}$ show the preferred orientation at (111) and the decrease of the current density to 25 or $10 \mathrm{~mA} / \mathrm{cm}^{2}$ results in the change of the preferred orientation to (200) or (220). The previous report suggest that the following reactions tend to occur during the

$$
\begin{aligned}
& \mathrm{H}_{2} \mathrm{O}+\mathrm{Ni} \rightarrow \mathrm{Ni}\left(\mathrm{H}_{2} \mathrm{O}\right) \\
& \mathrm{Ni}\left(\mathrm{H}_{2} \mathrm{O}\right) \rightarrow \mathrm{H}^{+}+\mathrm{Ni}(\mathrm{OH})^{+}+2 \mathrm{e}- \\
& \mathrm{OH}^{-}+\mathrm{Ni}(\mathrm{OH})^{+} \rightarrow \mathrm{Ni}(\mathrm{OH})_{2}
\end{aligned}
$$


electroforming process and cause the formation of $\mathrm{Ni}(\mathrm{OH})_{2}$ on the cathode surface [30]. As a result, the texture coefficient at the preferred orientation is noticeably decreased [30]. On the other hand, it is found for face-centred cubic metals, the stress in grains with orientation of (hkl) is linearly correlated with the angle between (100) and (hkl) [31]. Therefore, it might be feasible to roughly assess the residual stress within the electrodeposited Ni by determining the deviation of the specific (hkl) plane away from the (100) plane. The angles of (111), (200) and (220) planes to (100) plane can be calculated to be $54.7^{\circ}$, $0^{\circ}$ and $45^{\circ}$, respectively. The result suggests that in comparision with the $\mathrm{Ni}$ produced at a higher current density, a lower current density tends to cause the decrease of the stress in the deposited Ni. However, when a small current density is utilised, it takes a long time to complete the electroforming process and may cause the build-up of the stress."

\section{Conclusions}

In this study, electroforming was carried out to produce high precision micro $\mathrm{Ni}$ gears using a soft mould fabricated by photolithography and soft lithography techniques. Microstructures, phase compositions, densities and hardness of the gears electroformed at varied current densities were investigated. It is found surface roughness of the soft mould is similar to that of the master mould and a high precision replica is sucessfully produced. A gold layer deposited on the soft mould for the electrforming process is around 100nm thick and micro Ni gears electroformed display good shape profiles with feature size of as small as approximately $70 \mu \mathrm{m}$. SEM images indicate that a lower current density can lead to a smoother surface morphology and a finer microstructure of the $\mathrm{Ni}$. Uniform thickness and surface morphologies of the electroformed samples can be achieved using a low current density of $10 \mathrm{~mA} / \mathrm{cm}^{2}$. XRD results imply grain sizes of the Ni can be tuned by changing the current densities and when the current density is decreased from 50 to $10 \mathrm{~mA} / \mathrm{cm}^{2}$, the grain sizes of the $\mathrm{Ni}$ are found to decrease from around 50 to $30 \mathrm{~nm}$. Densities of the gears are closely associated with the current density, which determines the deposition rate of the $\mathrm{Ni}$ ions and the release of the bubbles during the electroforming process. Varied current densities cause the change of preferred orientation from (111) to (200) or (220) and a lower current density tends to cause the decrease of the stress in the deposited $\mathrm{Ni}$. The presented study suggests that massive 
reproduction of metallic micro components with improved mechanical properties might to be realized using the combined techniques.

\section{Acknowledgments}

This publication is based on the research supported by the European Horizon 2020 under grant number 644971, 111 Programme under grant number B12016 and Innovative UK project under grant number 710705.

\section{References}

1. Imbaby, M. F.; Jiang, K., Stainless steel-titania composite micro gear fabricated by soft moulding and dispersing technique. Microelectronic Engineering 2010, 87 (5-8), 1650-1654.

2. Liu, Q. Y.; Zhang, Q. H.; Zhang, M.; Zhang, J. H., Effect of Crystallographic Anisotropy on Micro EDM Process. Materials And Manufacturing Processes 2015, 30 (8), 961-967.

3. Li, L.; Li, Z. Y.; Wei, X. T.; Cheng, X., Machining Characteristics of Inconel 718 by Sinking-EDM and Wire-EDM. Materials And Manufacturing Processes 2015, 30 (8), 968-973.

4. Abdullahi, A. A.; Choudhury, I. A.; Azuddin, M., Process Development and Product Quality of Micro-Metal Powder Injection Molding. Materials And Manufacturing Processes 2015, 30 (11), 1377-1390.

5. Lee, S. Y.; Jiang, C. P., Development of a Three-Dimensional Slurry Printing System Using Dynamic Mask Projection for Fabricating Zirconia Dental Implants. Materials And Manufacturing Processes 2015, 30 (12), 1498-1504.

6. Huang, K. L.; Yang, Y.; Qin, Y.; Yang, G., 316L Stainless Steel Powder Densification during the Coupled Multi-Fields Activated Micro-Forming. Materials And Manufacturing Processes 2013, 28 (2), 183-188.

7. Lu, Z. L.; Lu, F.; Cao, J. W.; Li, D. C., Manufacturing Properties of Turbine Blades of Carbon Fiber-Reinforced SiC Composite Based on Stereolithography. Materials And Manufacturing Processes 2014, 29 (2), 201-209.

8. Imbaby, M.; Jiang, K.; Chang, I., Fabrication of 316-L stainless steel micro components using encapsulating soft mould and isopressing technique. Microelectronic Engineering 2010, 87 (5-8), $1623-1628$.

9. Mappes, T.; Worgull, M.; Heckele, M.; Mohr, J., Submicron polymer structures with X-ray lithography and hot embossing. Microsystem Technologies 2008, 14 (9-11), 1721-1725. 
10. Becker, E. W.; Ehrfeld, W.; Hagmann, P.; Maner, A.; Münchmeyer, D., Fabrication of microstructures with high aspect ratios and great structural heights by synchrotron radiation lithography, galvanoforming, and plastic moulding (LIGA process). Microelectronic Engineering 1986, $4(1), 35-56$.

11. Jafferson, J. M.; Hariharan, P., Investigation of the Quality of Microholes Machined by mu EDM Using Image Processing. Materials And Manufacturing Processes 2013, 28 (12), 1356-1360.

12. Muthuramalingam, T.; Mohan, B., Influence of Discharge Current Pulse on Machinability in Electrical Discharge Machining. Materials And Manufacturing Processes 2013, 28 (4), 375-380.

13. Wang, F.; Liu, Y. H.; Shen, Y.; Ji, R. J.; Tang, Z. M.; Zhang, Y. Z., Machining Performance of Inconel 718 Using High Current Density Electrical Discharge Milling. Materials And Manufacturing Processes 2013, 28 (10), 1147-1152.

14. Gietzelt, T.; Jacobi, O.; Piotter, V.; Ruprecht, R.; Hausselt, J., Development of a micro annular gear pump by micro powder injection molding. Journal Of Materials Science 2004, 39 (6), 2113-2119. 15. Ye, H.; Liu, X. Y.; Hong, H., Fabrication of metal matrix composites by metal injection molding-A review. Journal Of Materials Processing Technology 2008, 200 (1-3), 12-24.

16. Kuram, E.; Timur, G.; Ozcelik, B.; Yilmaz, F., Influences of Injection Conditions on Strength Properties of Recycled and Virgin PBT/PC/ABS. Materials And Manufacturing Processes 2014, 29 (10), 1260-1268.

17. Matschuk, M.; Larsen, N. B., Injection molding of high aspect ratio sub-100 nm nanostructures. Journal Of Micromechanics And Microengineering 2013, 23 (2).

18. Lee, C. H.; Jiang, K., Fabrication of thick electroforming micro mould using a KMPR negative tone photoresist. Journal Of Micromechanics And Microengineering 2008, 18 (5), 055032.

19. Wei, X. Y.; Zhu, Z. G.; Prewett, P. D.; Jiang, K., Fabrication of Ni-Al2O3 composite microcomponent by electroforming. Microelectronic Engineering 2007, 84 (5-8), 1256-1259.

20. Zhu, Z.; Wei, X.; Jiang, K., A net-shape fabrication process of alumina micro-components using a soft lithography technique. Journal Of Micromechanics And Microengineering 2007, 17 (2), 193.

21. Kim, J. S.; Jiang, K.; Chang, I., Pressure Free Fabrication of 3D Microcomponents Using Al Powder. Advanced Engineering Materials 2006, 8 (1-2), 38-41.

22. Hassanin, H.; Jiang, K., Optimized process for the fabrication of zirconia micro parts. Microelectronic Engineering 2010, 87 (5-8), 1617-1619. 
23. Zhu, Z.; Hassanin, H.; Jiang, K., A soft moulding process for manufacture of net-shape ceramic microcomponents. The International Journal of Advanced Manufacturing Technology 2010, 47 (1-4), 147-152.

24. Walczak, R.; Sniadek, P.; Dziuban, J. A., SU-8 photoresist as material of optical passive components integrated with analytical microsystems for real-time polymerase chain reaction. Optica Applicata 2011, 41 (4), 873-884.

25 Hili, K;Fan, D; Guzenko, V.A; Ekinci, Y., Nickel electroplating for high-resolution nanostructures. Microelectronic Engineering 2015, 141, 122-128.

26. van Huis, M. A.; Fedorov, A. V.; van Veen, A.; Falub, C. V.; Eijt, S. W. H.; Kooi, B. J.; De Hosson, J. T. M.; Hibma, T.; Zimmerman, R. L., Structural properties of Au and Ag nanoclusters embedded in MgO. Nuclear Instruments \& Methods in Physics Research Section B-Beam Interactions with Materials and Atoms 2002, 191, 442-446.

27. Ramalingam, S.; Muralidharan, V. S.; Subramania, A., Electrodeposition and characterization of $\mathrm{Cu}-\mathrm{TiO}(2)$ nanocomposite coatings. Journal of Solid State Electrochemistry 2009, 13 (11), 1777-1783.

28. Muresan, L.; Oniciu, L.; Froment, M.; Maurin, G., Inhibition of lead electrocrystallization by organic additives. Electrochimica Acta 1992, 37 (12), 2249-2254.

29. Chen, L.; Wang, L.; Zeng, Z.; Xu, T., Influence of pulse frequency on the microstructure and wear resistance of electrodeposited $\mathrm{Ni}-\mathrm{Al} 2 \mathrm{O} 3$ composite coatings. Surface and Coatings Technology 2006, $201(3-4), 599-605$.

30. Qu, N. S.; Zhu, D.; Chan, K. C., Fabrication of Ni-CeO2 nanocomposite by electrodeposition. Scripta Materialia 2006, 54 (7), 1421-1425.

31. Zhang, J.-M.; Zhang, Y.; Xu, K.-W., Anisotropy analysis of the thermal stresses and strain energies in BCC metal films. Physica B: Condensed Matter 2005, 368 (1-4), 215-222.

\section{Figures and tables}




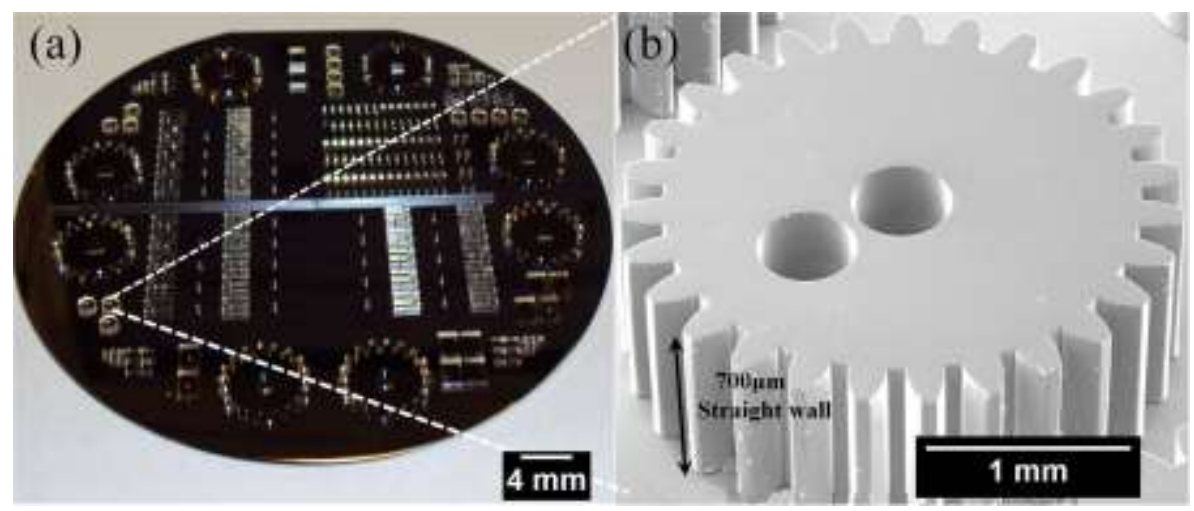

Figure 1.A camera image of the SU-8 ultrathick master mould on a silicon wafer (a) and a SEM image of the micro gear master mould (b).

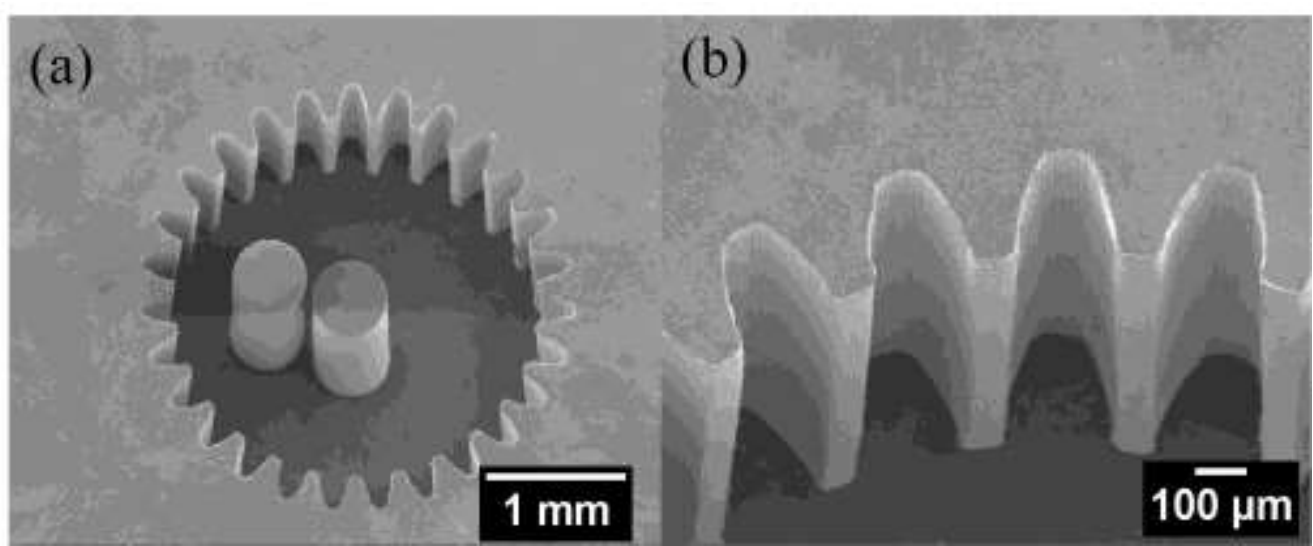

Figure 2. SEM images of the soft moulds of the gears. (b) is a higher magnification image of (a).

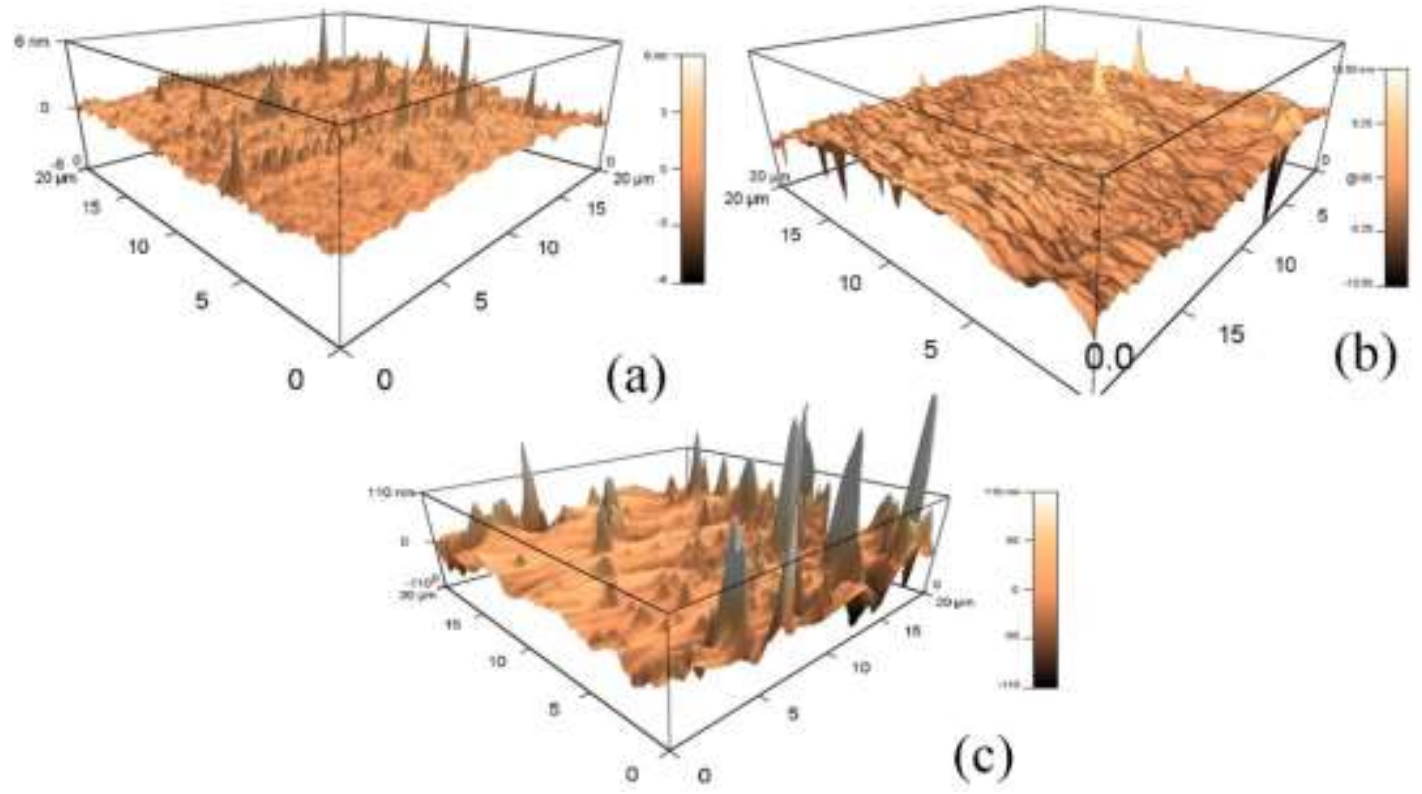

Figure 3. AFM images of the surfaces of the master mould (a), soft mould (b) and the gold-coated soft mould (c) 


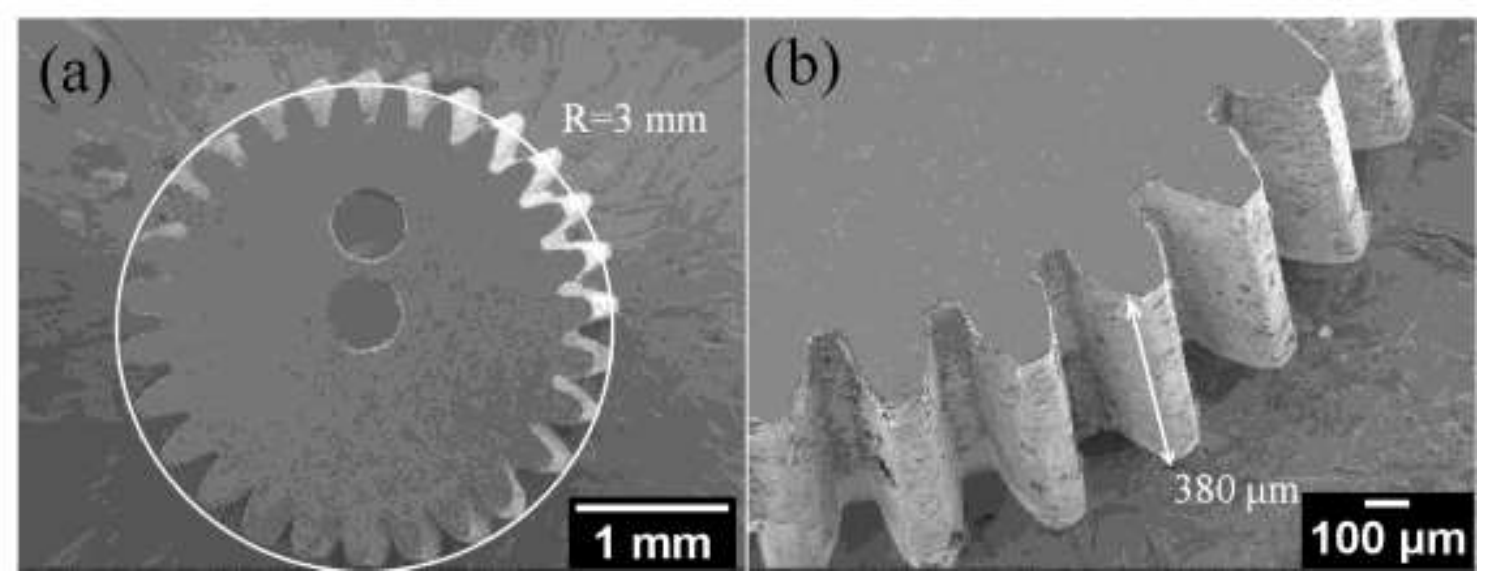

Figure 4. SEM images of the electroformed micro gears. (b) is a higher magnification image of (a). 

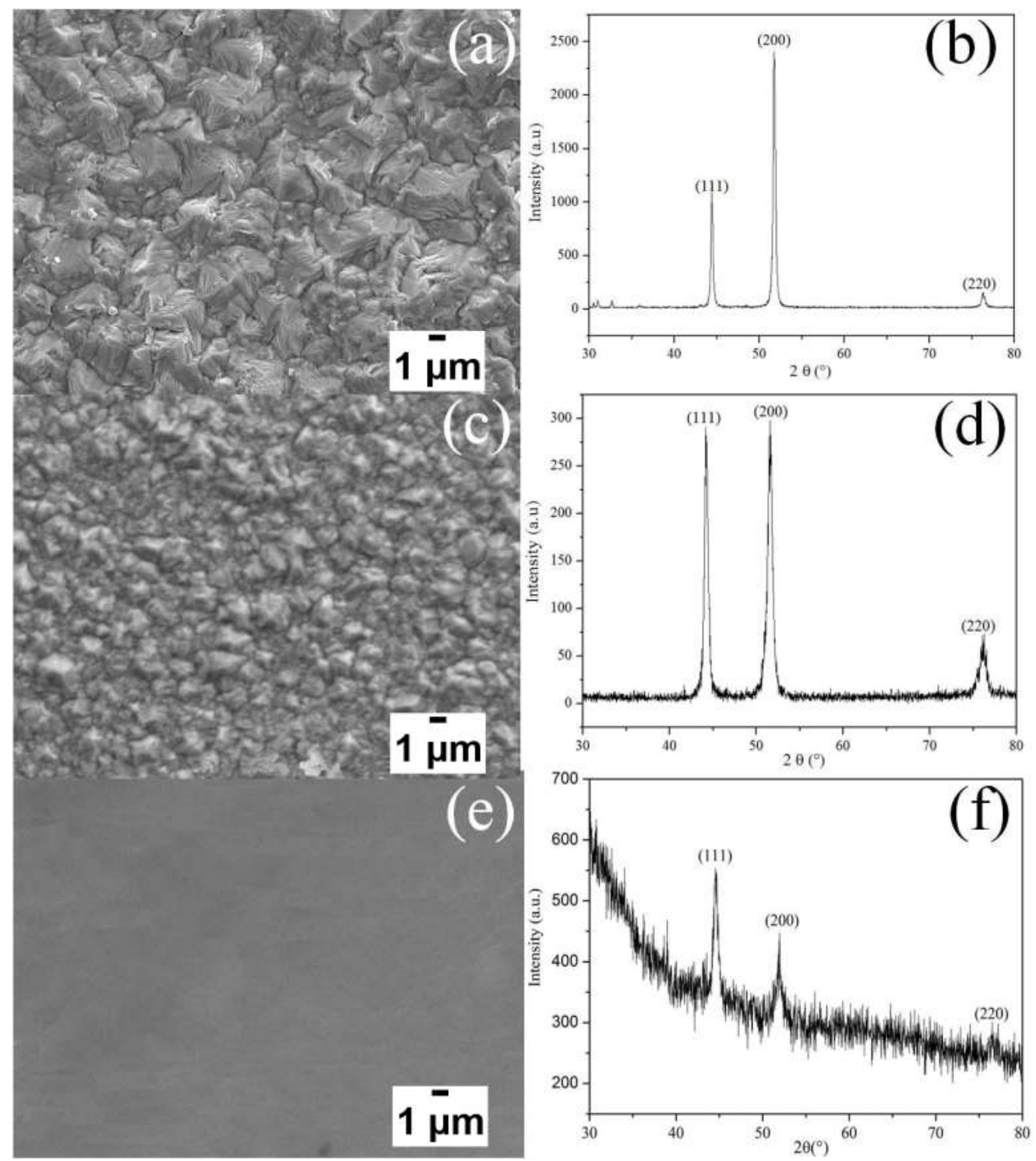

Figure 5 SEM and XRD results of the micro gears prepared at $50(a, b), 25(\mathrm{c}, \mathrm{d})$ and $10(\mathrm{e}, \mathrm{f}) \mathrm{mA} / \mathrm{cm}^{2}$ 


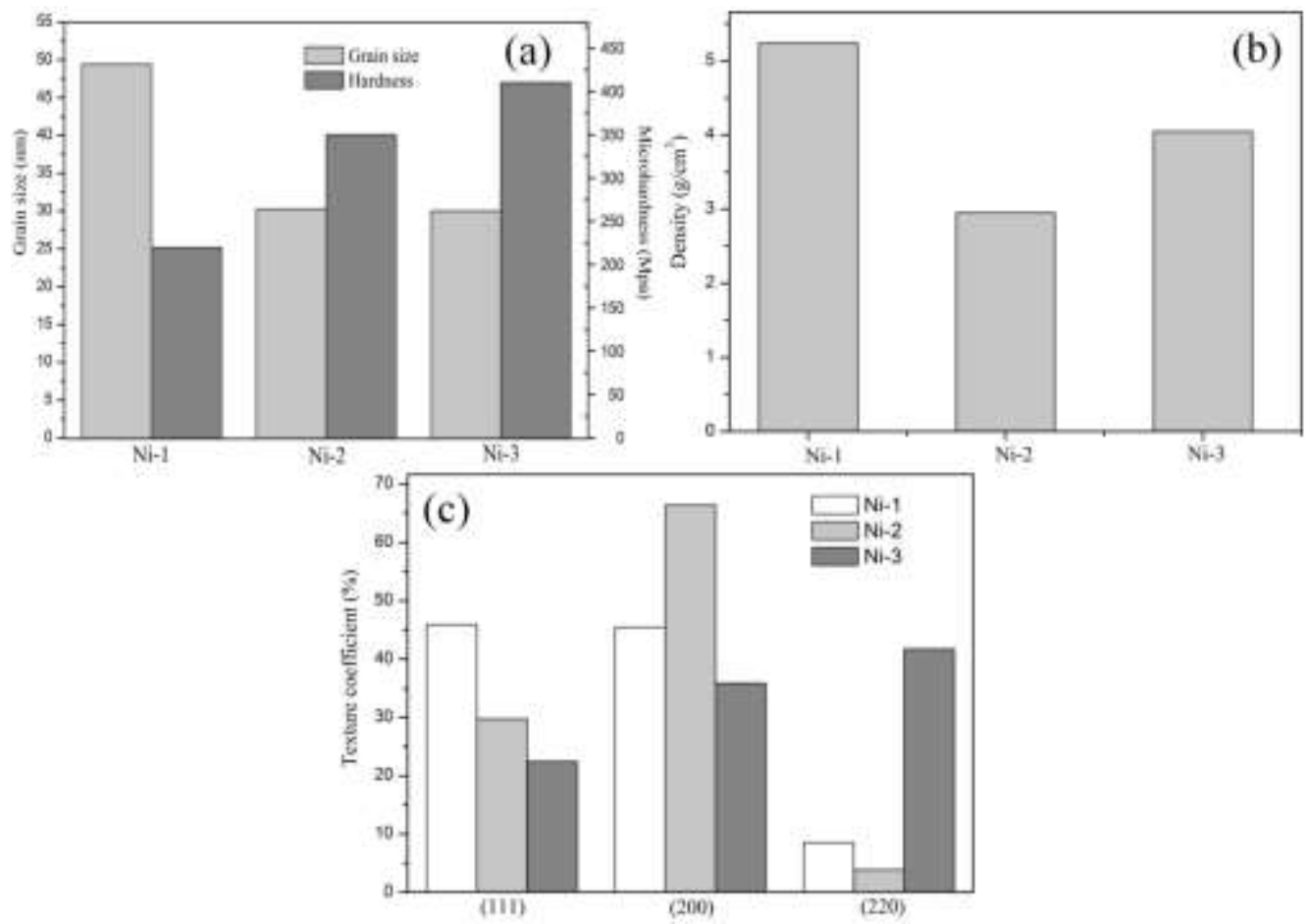

Figure 6. Hardness, grain sizes (a), densities (b) and texture coefficients(c) of the samples electroformed at varied current densities. 